\title{
INCLINED SURFACE CRACKS IN A GRADED HALF-PLANE SUBJECTED TO FRICTIONAL SLIDING CONTACT
}

\author{
Onur ArsLan \\ Eskisehir Osmangazi University, Department of Mechanical Engineering, Eskisehir, Turkey \\ e-mail: oarslan@ogu.edu.tr
}

\begin{abstract}
Fracture parameters of an inclined surface crack in a graded half-plane subjected to sliding frictional contact are investigated in this study. The problem is modelled via the finite element method (FEM) under the plane strain assumption employing a newly developed displacement boundary condition. The shear modulus of the half-plane is graded exponentially through the thickness direction by means of the homogeneous finite element approach. The augmented Lagrange algorithm is selected as an iterative contact solver. The mixed mode stress intensity factors (SIFs) which are induced by the contact stresses are evaluated utilizing the Displacement Correlation Technique (DCT). The accuracy of the present procedure is ensured comparing the SIF results to those calculated by an analytical method for vertical surface cracks. Additional SIF curves are generated as functions of the crack inclination in order to reveal the effects of non-homogeneity, punch location, crack length and friction coefficient. The prominent conclusion of this study indicates that the crack orientation has profound effects on the behavior of SIF curves, which has not been presented in any study published so far. Hence, consideration of crack inclination is proved essential to successfully predict fracture behavior of a graded medium under frictional contact.
\end{abstract}

Keywords: inclined surface cracks, crack/contact problems, graded materials, finite element method

\section{Introduction}

In engineering applications, keeping the structural integrity of a member is the major concern to extend service life. From this point of view, investigation of contact and fracture mechanics problems come into prominence especially for load transfer members. Brittle materials such as ceramics are well suited for load carrying surfaces due to their high wear resistance. However, because of their limited toughness they are prone to surface cracking under frictional contact loadings, which may lead to loss in fatigue strength (Hills et al., 1993). Such destructive effects of contact loadings can be partially tolerated by employing graded materials which consist of spatially varying composition of brittle and ductile materials. For instance, frictional contact and indentation related damages is shown to be mitigated upon introducing material gradation (Surresh, 2001). It is revealed that non-homogeneous ceramics are more damage resistant to loadings induced by spherical intenders (Lawn, 1998). Spatial gradation of Young's modulus is proven to prevent formation of Hertzian cone-cracks on brittle surfaces loaded by spherical stamps (Jitcharoen et al., 1998). Material gradation provides an improvement in damage tolerance of ceramics which are subjected to contact and flexural loadings (Zhang, 2012).

In literature, there are some computational and analytical studies analyzing behavior of cracks in elastic continua which are subjected to contact loadings. Hasebe et al. (1989) investigated edge crack problems in an elastic half plane under frictional contact by utilizing a rational mapping function and complex stress functions. Fracture parameters of a surface crack in a 
graded medium were evaluated under the action of frictional contact loadings by using the singular integral equation approach (Dag, 2001; Dag and Erdogan, 2002). Embedded horizontal cracks in isotropic (El-Borgi et al., 2004) and orthotropic (El-Borgi et al., 2013) graded coatings subjected to frictional Hertzian contact assumption were examined by means of the singular integral equation approach. An edge crack in a graded coating loaded by a flat rigid stamp was investigated by means of an uncoupled solution procedure based on the singular integral equation approach (Dag et al., 2012). Sarikaya and Dag (2016) analytically calculated fracture parameters of a surface crack in an orthotropic half-plane whose surface was exposed to sliding frictional contact loadings.

In all the aforementioned studies, the cracks are located parallel or perpendicular to the contact surface. However, propagation of the cracks is observed to be slanted in an elastic medium which is subjected to fretting contact (Faanes, 1995; Giner et al., 2014). Moreover, surface cracks may possibly initiate and propagate in any direction through a medium which is under non-symmetrical loading conditions, such as frictional contact loadings. Hence, effects of crack orientation on fracture parameters should be examined to clearly predict critical and sub-critical crack growth. The literature survey revealed that no computational or analytical studies related to inclined surface cracks in a graded medium under frictional contact has been conducted.

In this study, fracture parameters of an inclined surface crack in an elastic graded medium which is subjected to frictional sliding contact are investigated. The medium is modelled on the plane strain assumption by means of a finite element analysis software ANSYS (2013). A newly developed displacement boundary condition is imposed on the model to be able to improve the simulation capability. The shear modulus of the half-plane is graded exponentially through the thickness direction utilizing the homogeneous finite element method. In the solution of the nonlinear contact problem, the augmented Lagrange algorithm is selected. The crack tip SIFs induced from the contact stresses are calculated by using DCT. Comparisons of the results to those generated by the analytical method for vertical surface cracks (Dag, 2001) validate the accuracy of the proposed finite element procedure. Extra SIF curves are depicted as functions of crack inclination for different non-homogeneity parameters, punch location, relative crack length and friction coefficient. The striking outcome of this study indicates that the change in crack inclination have remarkable influence on the mixed mode SIFs. As a result, consideration of crack inclination in crack-contact problems is proved essential to successfully predict fracture behavior of a graded medium under frictional contact loadings.

\section{Problem definition and solution method}

The geometry of an inclined surface crack in a graded medium which is subjected to frictional sliding contact is depicted in Fig. 1. The surface of the graded medium is loaded by a rigid flat punch whose end points are located at $y=b$ and $y=c$. Coulomb's dry friction is assumed to exist between the rigid punch and the medium surface. Exponential shear modulus gradation is introduced through the thickness direction considering Poisson's ratio as a constant (Dag, 2001; Dag and Erdogan, 2002)

$$
\mu(x)=\mu_{0} \exp (\gamma x)
$$

where $\mu_{0}$ and $\gamma$ represent the shear modulus at the surface $(x=0)$ and the non-homogeneity parameter, respectively. Thus, constitutive relations for the graded medium can be stated as

$$
\varepsilon=\exp (-\gamma x) \mathbf{S} \boldsymbol{\sigma}
$$




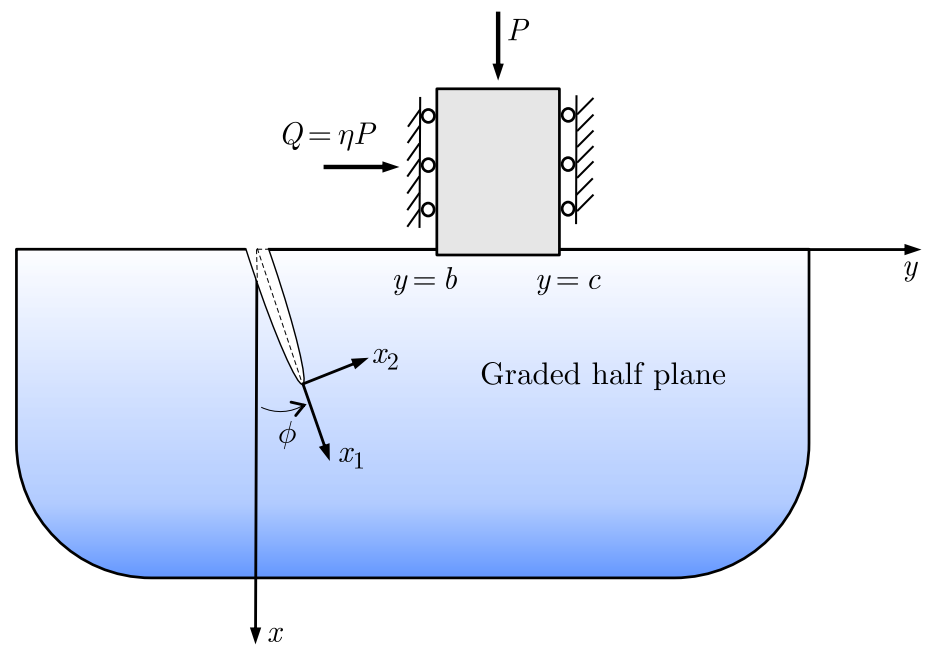

Fig. 1. Problem configuration

where $\mathbf{S}, \boldsymbol{\varepsilon}$ and $\boldsymbol{\sigma}$ respectively stand for the compliance matrix, strain vector and stress vector in the global coordinate system $x y$. The stress and strain vectors can be written explicitly as

$$
\varepsilon=\left[\varepsilon_{x x}, \varepsilon_{y y}, \varepsilon_{z z}, \varepsilon_{y z}, \varepsilon_{x z}, \varepsilon_{x y}\right]^{\mathrm{T}} \quad \boldsymbol{\sigma}=\left[\sigma_{x x}, \sigma_{y y}, \sigma_{z z}, \sigma_{y z}, \sigma_{x z}, \sigma_{x y}\right]^{\mathrm{T}}
$$

Isotropic materials have an infinite number of elastic symmetry planes and possess two independent material properties. The compliance matrix in Eq. (2.2) can be expressed in terms of the shear modulus $\mu_{0}$ and Poisson's ratio $\nu$ as follows (Jones, 1998)

$$
\mathbf{S}=\left[\begin{array}{cccccc}
C_{1} & C_{2} & C_{2} & 0 & 0 & 0 \\
C_{2} & C_{1} & C_{2} & 0 & 0 & 0 \\
C_{2} & C_{2} & C_{1} & 0 & 0 & 0 \\
0 & 0 & 0 & C_{1}-C_{2} & 0 & 0 \\
0 & 0 & 0 & 0 & C_{1}-C_{2} & 0 \\
0 & 0 & 0 & 0 & 0 & C_{1}-C_{2}
\end{array}\right]
$$

where

$$
C_{1}=\frac{1}{2(1+\nu) \mu_{0}} \quad C_{2}=-\frac{v}{2(1+\nu) \mu_{0}}
$$

Constitutive relations can be restated for plane elasticity in the local coordinate system $x_{1} x_{2}$ which is defined at the crack tip

$$
\left\{\begin{array}{c}
\varepsilon_{11} \\
\varepsilon_{22} \\
2 \varepsilon_{12}
\end{array}\right\}=\exp \left[-\gamma\left(x_{1}+a\right) \cos \phi\right]\left[\begin{array}{lll}
s_{11} & s_{12} & s_{16} \\
s_{21} & s_{22} & s_{26} \\
s_{61} & s_{62} & s_{66}
\end{array}\right]\left\{\begin{array}{c}
\sigma_{11} \\
\sigma_{22} \\
\sigma_{12}
\end{array}\right\}
$$

where $a, \phi$ and $s_{i j}(i, j=1,2,6)$ represent length of the surface crack, crack inclination angle and compliance coefficients, respectively. The compliance coefficients for the plane stress case read

$$
\begin{array}{ll}
s_{11}=s_{22}=C_{1} & s_{12}=C_{2} \\
s_{16}=s_{26}=0 & s_{66}=2\left(C_{1}-C_{2}\right)
\end{array}
$$

The compliance coefficients for the plane strain case are defined below

$$
\begin{array}{llrl}
s_{11} & =s_{22}=\frac{C_{1}^{2}-C_{2}^{2}}{C_{1}} & s_{12} & =\frac{C_{2}\left(1-C_{2}\right)}{C_{1}} \\
s_{16} & =s_{26}=0 & s_{66} & =2\left(C_{1}-C_{2}\right)
\end{array}
$$


Analytical expressions of the opening mode (mode I) and the shearing mode (mode II) SIFs are given as follows (Perez, 2004)

$$
K_{I}=\lim _{x_{1} \rightarrow 0^{+}} \sqrt{2 \pi\left(x_{1}-a\right)} \sigma_{22}\left(x_{1}, 0\right) \quad K_{I I}=\lim _{x_{1} \rightarrow 0^{+}} \sqrt{2 \pi\left(x_{1}-a\right)} \sigma_{12}\left(x_{1}, 0\right)
$$

In order to calculate SIFs through Eq. (2.8), one should determine the asymptotic stress expressions by conducting a heavy solution procedure based on the singular integral equation approach (Dag, 2001; Dag and Erdogan, 2002). On the other hand, SIFs can be calculated via DCT with a high level of accuracy within a properly constructed finite element model. DCT utilizes nodal displacements around crack tip which are computed through FEM (Kim and Paulino, 2003)

$$
K_{I}=\frac{1}{8} \sqrt{\frac{2 \pi}{\Delta a}} \frac{4 V_{1}-V_{2}}{s_{22} \exp [-\gamma a \cos \phi]} \quad K_{I I}=\frac{1}{8} \sqrt{\frac{2 \pi}{\Delta a}} \frac{4 U_{1}-U_{2}}{s_{11} \exp [-\gamma a \cos \phi]}
$$

where

$$
\begin{array}{ll}
U_{1}=u_{1}\left(N_{1}\right)-u_{1}\left(N_{3}\right) & U_{2}=u_{1}\left(N_{2}\right)-u_{1}\left(N_{4}\right) \\
V_{1}=u_{2}\left(N_{1}\right)-u_{2}\left(N_{3}\right) & V_{2}=u_{2}\left(N_{2}\right)-u_{2}\left(N_{4}\right)
\end{array}
$$

where $N_{j}(j=1, \ldots, 4)$ are the nodes around the crack tip, which are depicted in Fig. 2 . $\Delta a$ represents the characteristic length of the crack tip elements. $u_{1}$ and $u_{2}$ are the displacement components defined through $x_{1}$ and $x_{2}$ directions, respectively.

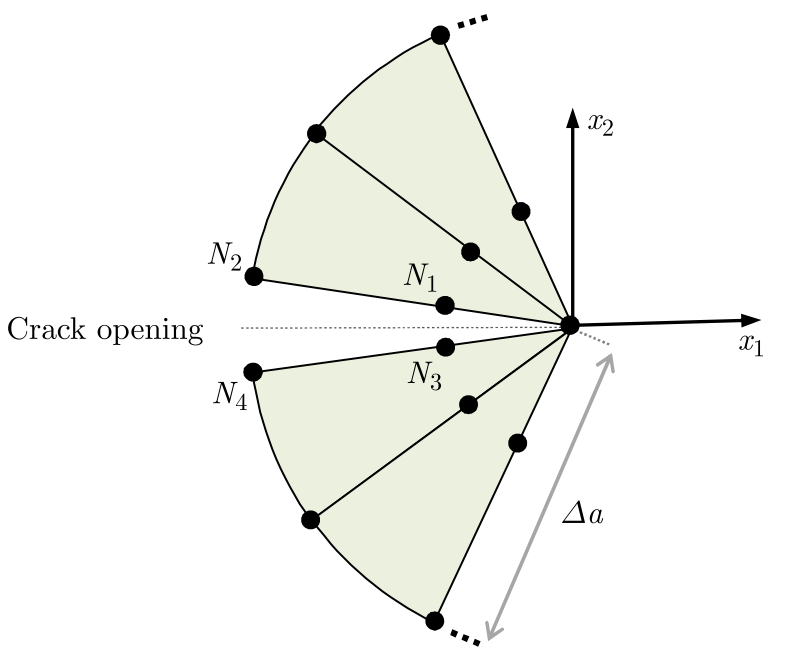

Fig. 2. Nodes on crack tip elements, $\Delta a / a=1 / 40000$

The finite element model (as seen in Fig. 3) possesses boundaries on the contrary to the original half-plane problem. $B, H$ and $W$ represent width of the flat punch, height of the model and width of the model, respectively. The ratios $B / H$ and $B / W$ are selected sufficiently small that they do not have any effect on the contact stresses and crack tip parameters.

When the side-lines (lines 1 and 2) of the model are set free, they experience some displacements under the effect of frictional contact stresses leading to incorrect crack tip parameters. These adverse displacement effects are observed to be still active, even if the model is chosen to be very large compared to the crack/contact region. Hence, the effect of side-line displacements on the crack-contact region should be neutralized in order to satisfactorily simulate the half-plane problem. If line 1 and line 2 are forced to undergo the same horizontal displacements under the effect of contact stresses, the behavior of the surface crack can be retrieved from the side-line effects to a certain degree. 


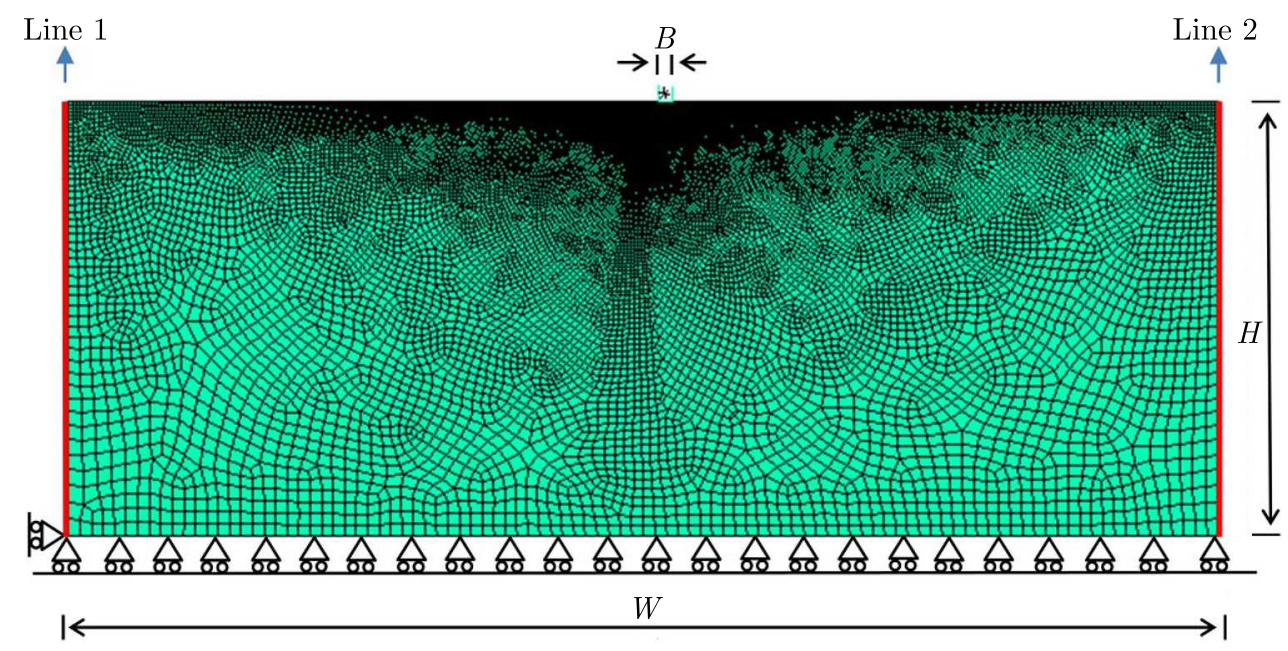

Fig. 3. Finite element model, $B / H=1 / 30, B / W=1 / 80, \phi=-30^{\circ}$

The following displacement boundary condition is imposed on the side-lines of the model which increases the simulation capability of the model

$$
v\left(x,-\frac{W}{2}\right)=v\left(x, \frac{W}{2}\right) \quad 0<x<H
$$

where $v(x, y)$ stands for the displacement component in the horizontal $y$-direction. To enable the boundary condition in Eq. (2.11), line 1 and line 2 are discretized with the same degree of mesh refinement. Hence, these lines acquire an equal number of nodes which are exactly at the same $x$-locations as illustrated in Fig. 4 . The $y$-component displacements of the nodes that locate at the same $x$-coordinates are equalized by inserting a subroutine into the ANSYS code. Thus Eq. (2.11) is treated in terms of the nodal displacements as

$$
v\left(N_{1 k}\right)=v\left(N_{2 k}\right) \quad k=1,2,3, \ldots, p
$$

where $N_{i k}$ represents the number of the nodes along line 1 and line 2 as depicted in Fig. 4. $p$ and $d_{k}$ respectively stand for the total number of the nodes and the vertical distances between consecutive nodes on each of line 1 and line 2.

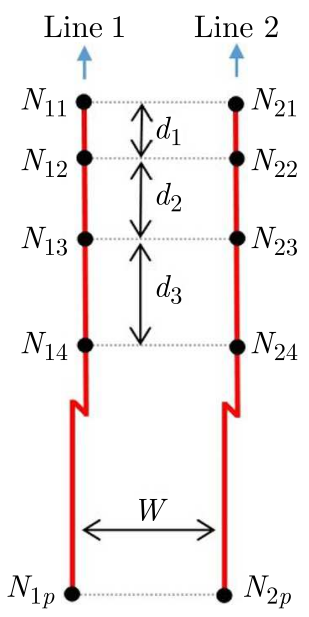

Fig. 4. Nodes on the side lines of the finite element model

As seen in Fig. 3, $x$-component displacement of the bottom line is restricted to zero, and the node located at the left-bottom corner is entirely fixed. 
The plane strain assumption is considered in the model which is meshed by using a total of 219030 8-noded quadrilateral and 6-noded triangular finite elements. Throughout the meshing operation, a 6 -noded triangular finite element is obtained by combining 3 nodes of an 8-noded quadrilateral finite element at a single point. The crack tip is discretized by using 48 regularly oriented triangular finite elements as shown in Fig. 5. The mid-side nodes of the crack tip elements (see Fig. 2) are positioned at a quarter of the radius of the crack tip elements. The crack tip SIFs are evaluated by means of Eq. (2.9) which makes use of nodal displacements around the crack tip.

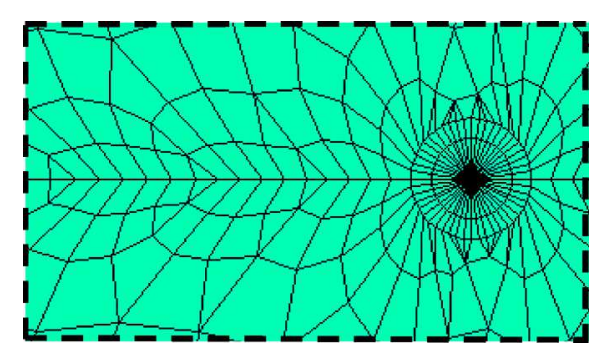

Fig. 5. Finite element mesh of the crack tip

Since contact stresses and contact surfaces are both unknown, contact mechanics problems involve a severe non-linearity. Due to this fact, finite element solutions of frictional contact problems are conducted by using step-by-step iterative procedures. The selected procedure in this study is the augmented Lagrange algorithm, in which consecutive equilibrium iterations are performed per increment of the normal and frictional forces. The details of the algorithm can be seen in the review paper by Mijar and Arora (2004). In the finite element model, three rigid target elements are used to represent rigid surfaces of the flat punch. The contact surface of the elastic medium is finely meshed using 300 contact line elements in order to get sharp variations of the contact stresses. The contact line elements are constructed by the three nodes of underlying solid elements (ANSYS, 2013). The Gauss integration points on the contact line elements are attributed as the contact detection points.

In the previous finite element studies related to graded structures, gradations of material properties through spatial coordinates are provided by using the graded finite element method and the homogeneous finite element method. In the graded finite element method (Santare and Lambros, 2000), the material properties are specified at the Gauss integration points of each finite element. However, in the homogeneous finite element method, the material properties are computed at the centroid. In the theoretical solutions for the contact and fracture mechanics problems of graded structures, utilization of the homogeneous finite element method yields highly accurate results (Dag et al., 2007, 2009; Arslan, 2017). Hence, in this study, the shear modulus gradation through the thickness direction is introduced by using the homogeneous finite element method.

\section{Numerical results}

In this Section, validity of the present procedure is revealed comparing the results to those generated by an analytical method (Dag, 2001) for a vertical surface crack $\left(\phi=0^{\circ}\right)$. Moreover, some curves of the mixed mode SIFs as functions of the crack inclination $\phi$ are presented for different problem parameters.

As depicted in Fig. 1, complete frictional contact occurs between the flat rigid stamp and the surface of a graded medium which involves an inclined surface crack. $\eta$ stands for the Coulomb friction coefficient prevailing between the FGM surface and the flat stamp. The locations $y=b$ 
and $y=c$ at the contact surface are called the trailing and leading ends of the contact zone, respectively.

Before going through the details of numerical results, it is beneficial to elaborate on the normalizations that are taken into account. The normalized non-homogeneity parameter, relative punch location and relative crack length are represented as $\gamma a, b / a, a /(c-b)$, respectively. Note that the elastic medium is homogeneous when $\gamma a=0$ and, shear modulus of the elastic medium increases in the positive $x$-direction when $\gamma a>0$. In addition, normalizations of mode I $\left(K_{I}\right)$ and mode II $\left(K_{I I}\right)$ SIFs at the crack tip are defined as

$$
K_{I n}=\sqrt{\frac{a}{\pi}} \frac{K_{I}}{P} \quad K_{I I n}=\sqrt{\frac{a}{\pi}} \frac{K_{I I}}{P}
$$

Note that negative mode I $\left(K_{I}\right)$ SIF results are not valid, and the negativity reveals that the crack closure occurs. However, these negative results are still useful and applicable in consideration of some problems including remote loading (Dag and Erdogan, 2002). Hence, in literature, negative $K_{I}$ results have been presented in parametric analysis of crack/contact problem solutions (Dag, 2001; Dag and Erdogan, 2002; Sarikaya and Dag, 2016).

Figure 6 reveals comparisons of the results to those evaluated analytically by Dag (2001) for vertical surface cracks $\left(\phi=0^{\circ}\right)$. The comparisons are depicted for the combinations of two different normalized non-homogeneity paremeters $\gamma a$ and four different friction coefficients $\eta$. As can be observed in this figure, excellent agreement between the analytical (Dag, 2001) and present results is attained in each case, which indicates the validity and reliability of the present finite element solution.

(a)

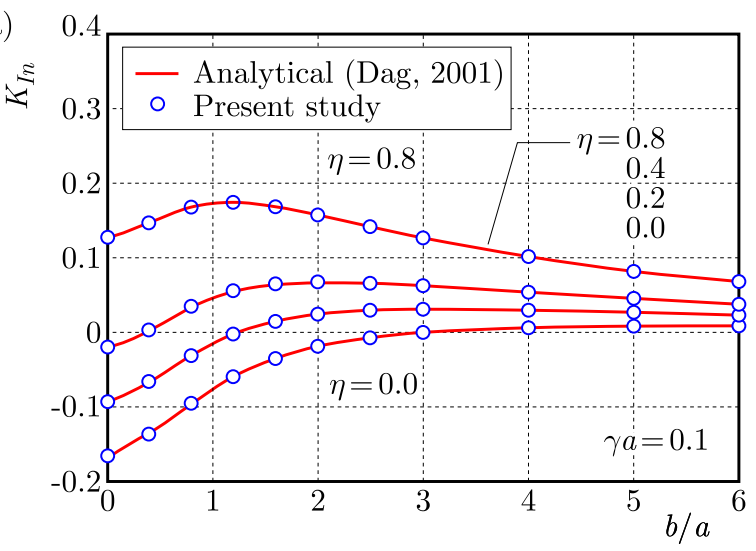

(c)

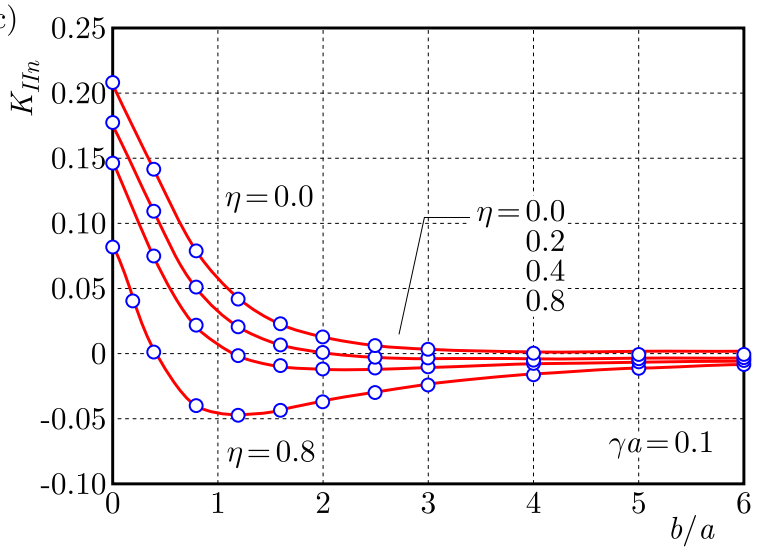

(b)

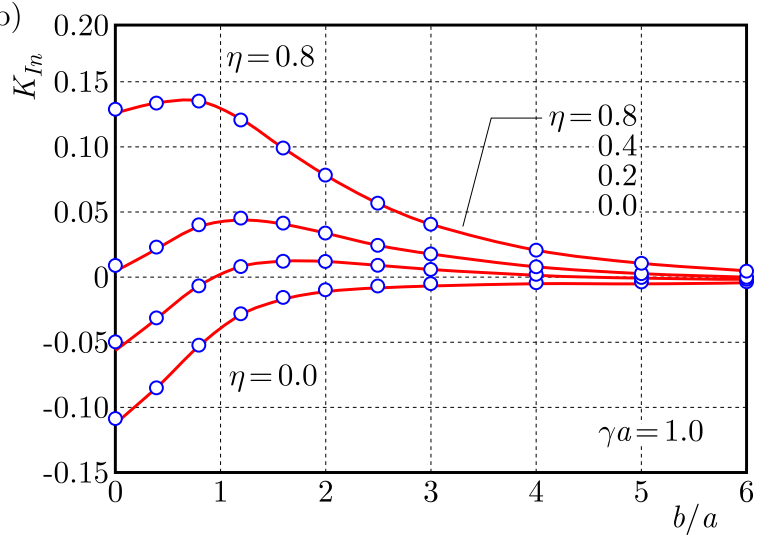

(d)

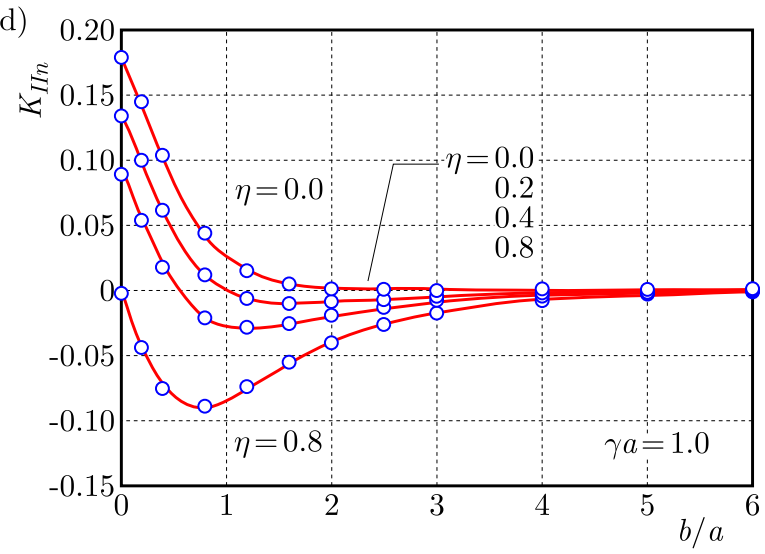

Fig. 6. Comparisons to the analytical results obtained by Dag (2001) for a graded isotropic half plane for $\nu=0.25, a /(c-b)=1.0, \phi=0^{\circ}$; (a) $K_{I n}, \gamma a=0.1$, (b) $K_{I n}, \gamma a=1.0$, (c) $K_{I I n}, \gamma a=0.1$,

(d) $K_{I I n}, \gamma a=1.0$ 
Figure 7 illustrates deformed shape of the graded medium for $a /(c-b)=1.0, b / a=1.0$, $\phi=-30^{\circ}, \eta=0.4, \nu=0.25$, and $\gamma a=1.0$. Mesh densities on the crack-contact region can be seen in this figure, and the surface crack is observed to be opened under the action of frictional contact loading. Figure 8 reveals the effect of normalized non-homogeneity parameter $\gamma a$ on the curves of normalized mixed-mode SIFs versus the inclination angle $\phi$. Examining Fig. 8a, when the normalized non-homogeneity parameter $\gamma a$ is increased from 0.1 to $1.0, K_{\text {In }}$ decreases for $\phi<0^{\circ}$, however increases for $\phi>0^{\circ}$. Note that the crack closure $\left(K_{I n}=0\right)$ occurs at higher inclination angles $\phi$, as $\gamma a$ is increased from 0.1 to 1.0. Observing Fig. 8b, when $\gamma a$ is increased from 0.1 to $1.0, K_{I I n}$ has an decreasing trend in the range $-20^{\circ}<\phi<60^{\circ}$. However, this trend is reversed in the range $-60^{\circ}<\phi<-20^{\circ}$. Also note that the absolute value of $K_{I I n}$ is critical in design, not its sign.

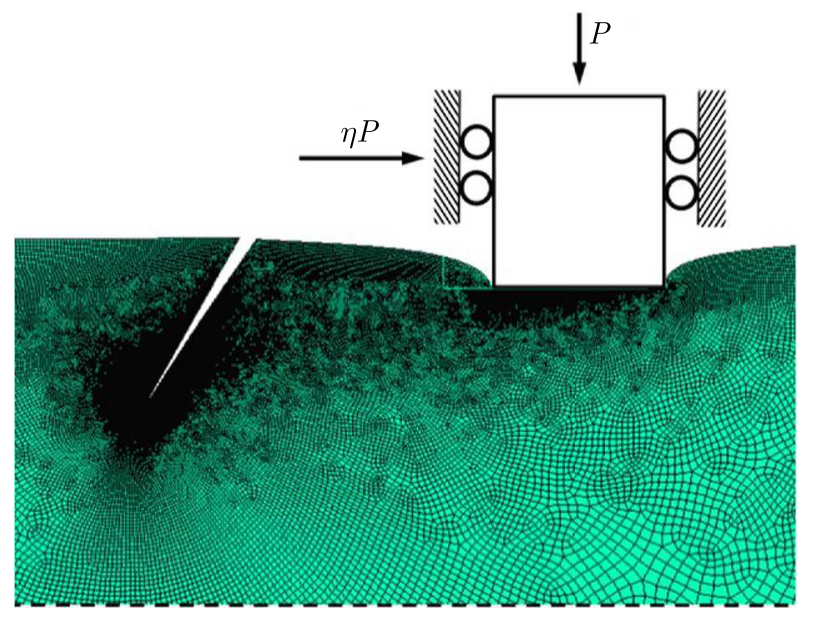

Fig. 7. Deformed shape of the crack-contact region, $\nu=0.25, a /(c-b)=1.0, b / a=1.0, \phi=-30^{\circ}$, $\eta=0.4, \gamma a=1.0$

(a)

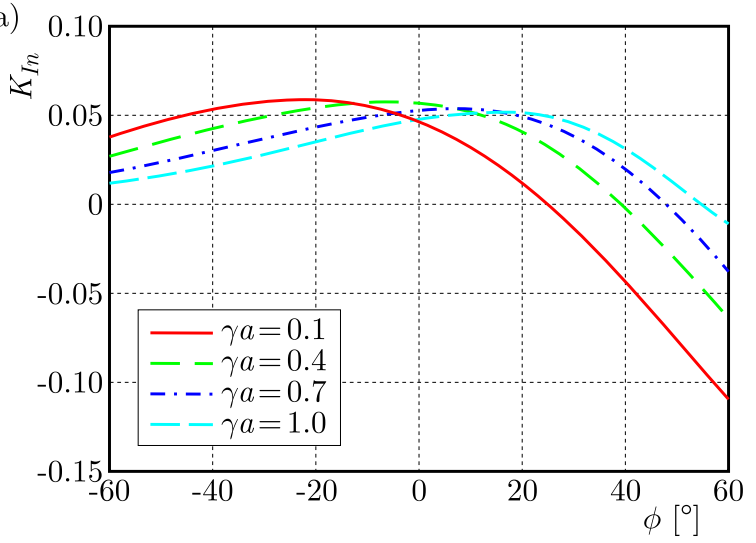

(b)

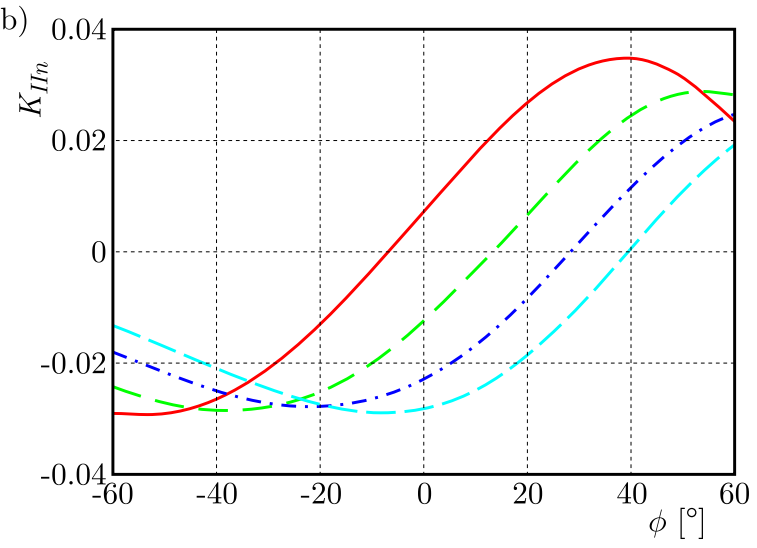

Fig. 8. Effect of normalized non-homogeneity parameter on normalized mode I (a) and mode II (b) SIFs, $\nu=0.25, a /(c-b)=1.0, b / a=1.0, \eta=0.4$

Figure 9 demonstrates the plots of normalized mixed-mode SIFs at the crack tip versus the inclination angle $\phi$ for different coefficients of friction $\eta$. When the coefficient of friction is increased from 0.0 to $0.8, K_{I n}$ elevates and $K_{I I n}$ decreases significantly. Note that the crack seems closed for $\eta<0.2$. In Fig. 10, the normalized mixed-mode SIFs at the crack tip are plotted versus the inclination angle $\phi$ for different relative crack lengths $a /(c-b)$. As seen in Fig. 10a, $K_{\text {In }}$ increases remarkably when $a /(c-b)$ is decreased from 5.0 to $0.6 \overline{6}$ for $\phi>0$. When $\phi<0$, $K_{I n}$ is not considerably affected by the change in $a /(c-b)$. Moreover, the crack closure seems to occur at higher inclanation angles $\phi$, as $a /(c-b)$ is decreased from 5.0 to $0.6 \overline{6}$. As displayed 
in Fig. 10b, when $a /(c-b)$ is decreased from 5.0 to $0.6 \overline{6}, K_{\text {IIn }}$ decreases for $\phi>0$ and elevates for $\phi<0$.

(a)

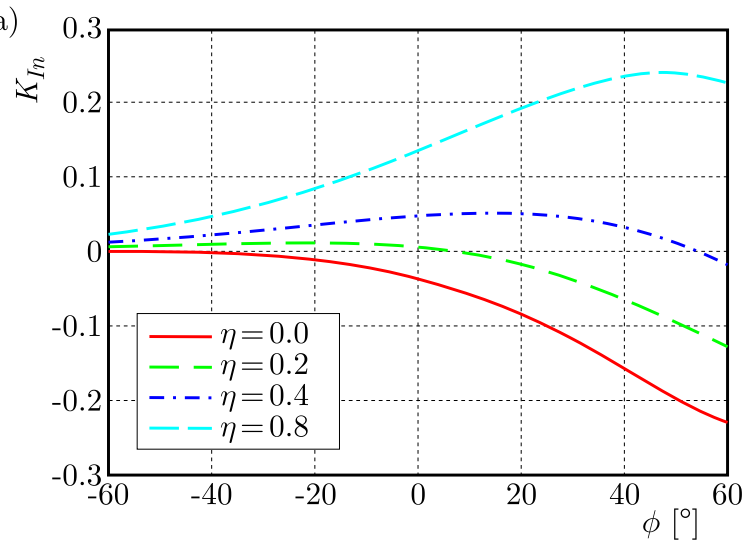

(b)

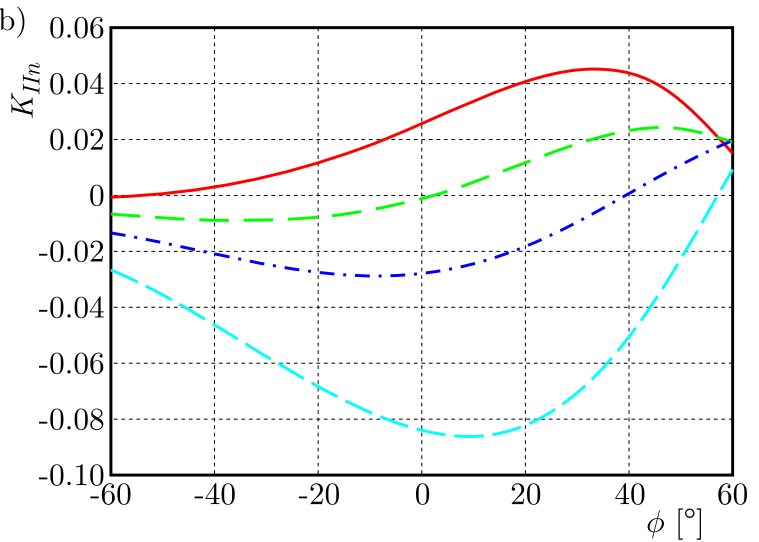

Fig. 9. Effect of friction on normalized mode I (a) and mode II (b) SIFs, $\nu=0.25, a /(c-b)=1.0$, $b / a=1.0, \gamma a=1.0$

(a)

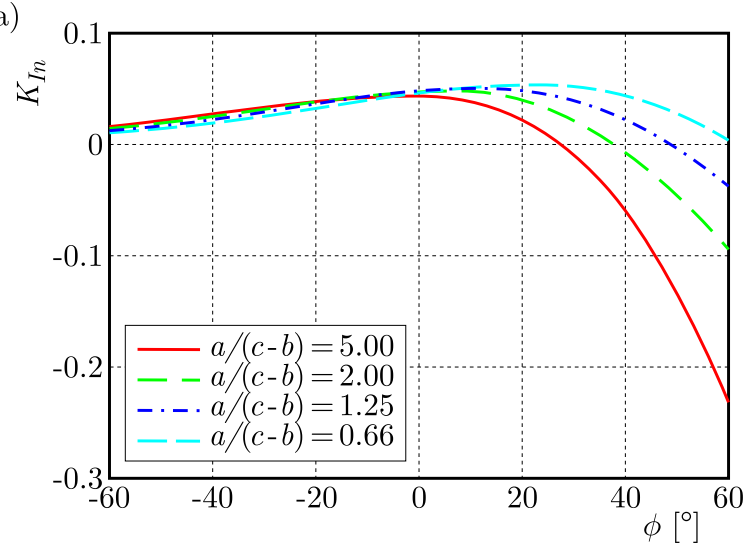

(b)

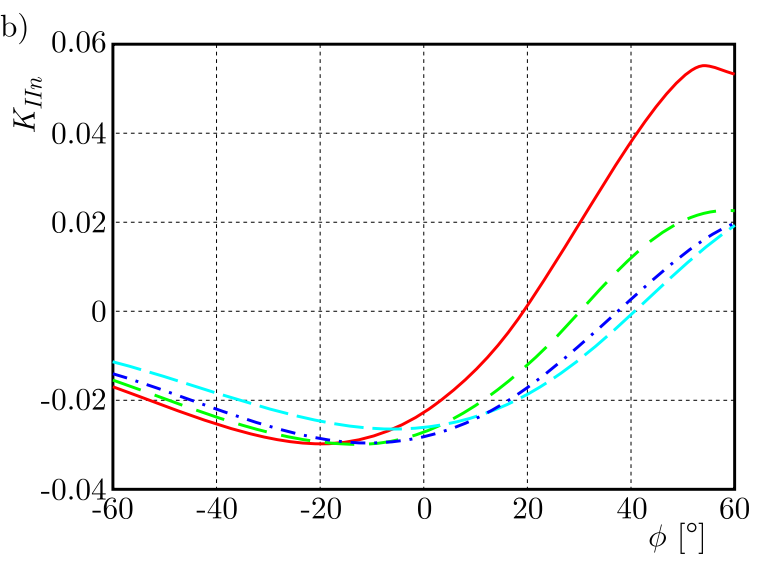

Fig. 10. Effect of relative crack length $a /(c-b)$ on normalized mode I (a) and mode II (b) SIFs, $\nu=0.25, b / a=1.0, \gamma a=1.0, \eta=0.4$

(a)

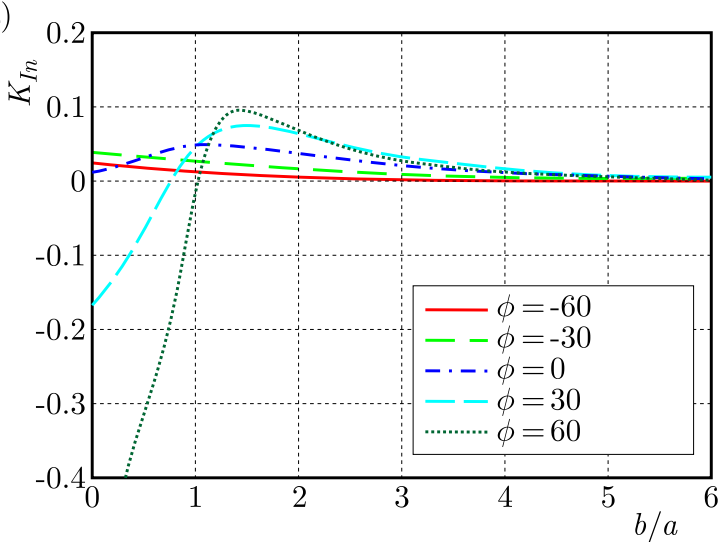

(b)

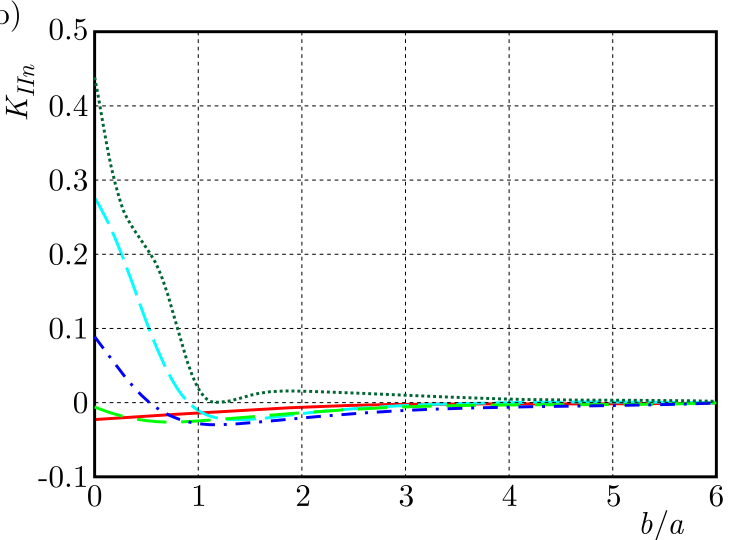

Fig. 11. Variations of normalized mode I (a) and mode II (b) SIFs versus the normalized punch location $b / a$ for different crack inclinations, $\nu=0.25, a /(c-b)=1.0, \gamma a=1.0, \eta=0.4$

Figure 11 illustrates the plots of normalized mixed-mode SIFs at the crack tip versus the normalized punch location $b / a$ for different crack inclanation angles $\phi$. As seen in Fig. 11a, $K_{\text {In }}$ reaches the local extremum when $b / a$ is between 1.0 and 2.0 for $0 \leqslant \phi \leqslant 60$. In this range 
of $b / a$, magnitude of $K_{I n}$ elevates significantly when the inclination angle $\phi$ is increased from $-60^{\circ}$ to $60^{\circ}$. It can be also infered from Fig. 11a that the crack is substantially closed $\left(K_{I n}<0\right)$ for $\phi \geqslant 30^{\circ}$ in the range $0<b / a<1.0$.

\section{Conclusions}

In literature, an analytical solution for a vertical surface crack problem in a graded medium subjected to frictional contact is available. In this study, the problem is extended computationally concerning different orientations of the surface crack to investigate fracture behavior of graded continua in a more insightful manner. The graded semi-infinite medium which is loaded by a flat rigid punch is simulated with a finite element model considering a particular displacement boundary condition. The plane strain assumption is taken into account in the model. The solutions of contact and crack problems are managed employing the augmented Lagrange algorithm and DCT, respectively. Comparisons of the results to those evaluated by an analytical technique for a vertical surface crack verify the accuracy of the present finite element approach. Further numerical results enlighten the behavior of the surface cracks for different problem parameters, which can be utilized efficiently in design and optimization. For instance, variation in material gradation has a changing effect on the crack tip parameters as the inclination angle $\phi$ alters. For the surface cracks possessing negative inclination $(\phi<0)$, the degree of gradation can be increased to suppress mode I type crack propagation. However, for surface cracks having positive inclination $(\phi>0)$, the risk of mode I type failure may arise as the degree of gradation is increased. The risk of mode I failure seems existing especially for $\eta>0.2$. For the surface cracks having negative inclination $(\phi<0)$, the risks of mode II type failure can be alleviated substantially by increasing the contact length. However, for the surface cracks having positive inclination $(\phi>0)$, the risk of mode I type crack propagation can be mitigated upon decreasing the contact length.

To conclude: effects of the problem parameters on the crack tip SIFs are shown to be altered significantly with the variation in crack orientation $\phi$, which is the main outcome of this study. Hence, investigation of the crack tip parameters only for vertical cracks is shown to be insufficient and, therefore, surface crack problems should be examined by taking the crack orientation into account to be able to clearly predict the critical and sub-critical surface crack growth in graded structures subjected to frictional contact loadings. A new displacement boundary condition is also proposed to improve the computational modelling of crack/contact problems.

\section{References}

1. ANSYS Inc., 2013, Ansys Basic Analysis Procedures Guide - Release 15, Ansys Inc., Canonsburg

2. Arslan O., 2017, Computational contact mechanics analysis of laterally graded orthotropic halfplanes, World Journal of Engineering, 14, 145-154

3. DAG S., 2001, Crack and contact problems in graded materials, Ph.D. Thesis, Lehigh University, Bethlehem

4. Dag S., Apatay T., Guler M.A., Gulgec M., 2012, A surface crack in a graded coating subjected to sliding frictional contact, Engineering Fracture Mechanics, 80, 72-91

5. Dag S., ERdogan F., 2002, A surface crack in a graded medium loaded by a sliding rigid stamp, Engineering Fracture Mechanics, 69, 1729-1751

6. Dag S., Guler M.A., Yildirim B., Ozatag A.C., 2009, Sliding frictional contact between a rigid punch and a laterally graded medium, International Journal of Solids and Structures, 46, 4038-4053 
7. Dag S., Yildirim B. And Sarikaya D., 2007, Mixed-mode fracture analysis of orthotropic functionally graded materials under mechanical and thermal loads, International Journal of Solids and Structures, 44, 7816-40

8. El-Borgi S., Keer L., Wissem B.S., 2004, An embedded crack in a functionally graded coating bonded to a homogeneous substrate under frictional Hertzian contact, Wear, 257, 760-776

9. El-Borgi S., Keer L., Wissem B.S., 2013, An embedded crack in a functionally graded orthotropic coating bonded to a homogeneous substrate under frictional Hertzian contact, International Journal of Solids and Structures, 50, 3898-3910

10. FaAnes S., 1995, Inclined cracks in fretting fatigue, Engineering Fracture Mechanics, 80, 6, 71-82

11. Giner E., Sabsabi M., Rodenas J.J., Fuenmayor F.J., 2014, Direction of crack propagation in a complete contact fretting-fatigue problem, International Journal of Fatigue, 58, 172-180

12. Hasebe N., Okumura M., Nakamura T., 1989, Frictional punch and crack in plane elasticity, Journal of Engineering Mechanics, 115, 6, 1137-1149

13. Hills D.A., Nowell D., SACKField A., 1993, Mechanics of Elastic Contacts, Butterworth-Heinemann, London

14. Jitcharoen J., Padture N.P., Giannakopoulos A.E., Suresh S., 1998, Hertzian-Crack suppression in ceramics with elastic-modulus-graded surfaces, Journal of the American Ceramic Society, 81, 9, 2301-2308

15. Jones R.M., 1998, Mechanics of Composite Materials, Taylor \& Francis, Philadelphia

16. Kim J-H., Paulino G.H., 2003, Mixed-mode J-integral formulation and implementation using graded elements for fracture analysis of nonhomogeneous orthotropic materials, Mechanics of Materials, 35, 107-128

17. LAWn B., 1998, Indentation of ceramics with spheres: a century after Hertz, Journal of the American Ceramic Society, 81, 8, 1977-1994

18. Mijar A.R., Arora J.S., 2004, An augmented Lagrangian optimization method for contact analysis problems, 1: formulation and algorithm, Structural Multidisciplinary Optimization, 28, 99-112

19. Perez N., 2004, Fracture Mechanics, Kluwer Academic Publishers, Boston

20. Santare M.H., Lambros J., 2000), Use of graded finite elements to model behavior of nonhomogeneous materials, Journal of Applied Mechanics, 67, 819-822

21. Sarikaya D., Dag S., 2016, Surface cracking in an orthotropic medium subjected to frictional contact, International Journal of Solids and Structures, 90, 1-11

22. Surresh S., 2001, Graded materials for resistance to contact deformation and damage, Science, 292, 5526, 2447-2451

23. ZHANG Y., 2012, Overview: damage resistance of graded ceramic restorative materials, Journal of the European Ceramic Society, 32, 11, 2623-2632 